\title{
Inovação aberta: uma vantagem competitiva para pequenas e médias empresas
}

\author{
Open Innovation: a competitive advantage for small and medium enterprises
}

\author{
Daniela Baggio ${ }^{1}$ \\ luri Gavronski ${ }^{2}$ \\ Vinicius Zanchet De Lima ${ }^{3}$
}

\begin{abstract}
Resumo
A dinâmica do mercado e a competição fazem com que as pequenas empresas desenvolvam ações e adotem estratégias para manterem uma posição almejada no mercado. Esta pesquisa teve por objetivo identificar como a inovação aberta foi relacionada à vantagem competitiva para as pequenas e médias empresas nos principais estudos existentes, como, também, prover uma agenda para futuras pesquisas. Assim, através de um levantamento bibliográfico em periódicos internacionais relevantes para essa área do conhecimento foram identificados os seguintes tópicos: vantagem competitiva nas organizações; pequenas e médias empresas no ambiente dos negócios; inovação aberta; e a inovação aberta: uma vantagem competitiva para pequenas e médias empresas. Com esse levantamento, verificou-se que a inovação aberta oferece um novo modelo de competição nas pequenas e médias empresas, além de trazer benefícios e vantagens excepcionais para a gestão. Dentre os benefícios, destacam-se: maior variedade de ideias e de conhecimento gerado; descoberta de soluções radicais; e legitimidade no ambiente externo. Além do mais, os parceiros externos podem ser mais bem equipados para fins de comercializar invenções para interesses mútuos. Torna-se possível o aproveitamento de complementos de terceiros, acesso a recursos e conhecimentos. Outros benefícios são custos mais baixos na inovação, riscos menores com as outras empresas. Explorar os recursos de outras empresas faz com que as pequenas empresas reduzam o risco de entrada no mercado e o tempo de desenvolvimento de um novo produto.
\end{abstract}

Palavras-chave: inovação aberta; vantagem competitiva; pequenas e médias empresas.

\begin{abstract}
The dynamics of the market and the competition between companies cause them to develop actions and adopt strategies to maintain a market-oriented position. This research aimed to identify how open innovation was related to the competitive advantage for small and medium enterprises in existing studies, as well as to provide an agenda for future research. Thus, through a theoretical bibliographical survey in newspapers and magazines relevant to this area of knowledge, we identifiedthe following topics: competitive advantage in organizations; small and medium enterprises in the business environment; open innovation; and open innovation: a competitive advantage for small and medium-sized enterprises. From this perspective, it is possible to perceive a new model of competition in small and medium-sized companies, in addition to bringing exceptional benefits and advantages to management, among them a great variety of ideas and generated knowledge, radical solutions discovery, legitimacy in the external environment, in addition, the external partners can be better equipped for the commercialization of inventions for mutual interests, besides the use of third-party complements, access to resources and knowledge. Other benefits are the prospect of lower costs in innovation, companies have a chance of reducing risks with other companies, exploiting the resources of other companies cause these to reduce the risk of market entry and the development time of a new product.
\end{abstract}

Keywords: Open innovation; competitive advantage; small and medium-sized enterprises.

Bacharelado em Administração de Empresas com linha de formação específica em Finanças e Controladoria (2013). Mestre em Administração pela Universidade do Vale do Rio dos Sinos (Unisinos) (2016). Afiliação: Universidade do vale do rio dos sinos. Brasil. Lattes: http://lattes.cnpq. br/3462904512356693 Email: baggiodaniela@gmail.com

2 Bolsista de pós-doutorado pela CAPES na Ivey Business School, Western University, Ontario, Canadá. Doutor em Administração pelo PPGA/ UFRGS (2009). Afiliação: Universidade do vale do rio dos sinos. Brasil. Lattes: http://lattes.cnpq.br/8843390959025944 Email: igavronski@ unisinos.br

3 Aluno do Doutorado em Administração na Universidade de Caxias do Sul, Bolsista PROSUP/ CAPES. Mestre em Administração pela Universidade de Caxias do Sul (2015). Afiliação: Universidade de Caxias do Sul. Brasil. Lattes: http://lattes.cnpq.br/7214657000873907 Email: vinizanchet@gmail.com 


\section{Introdução}

O ambiente atual de negócios é caracterizado pela necessidade das empresas operarem de forma conjunta, assim associando os recursos a partir da definição das estratégias da empresa. Por sua vez, as empresas precisam de processos inovadores para se manterem competitivas no mercado em que estão inseridas. Inovar é um desafio ainda maior para empresas de pequeno e médio porte, pois possuem mais limitações de recursos financeiros e capacidades do que grandes empresas.

A inovação é um elemento fundamental para a competitividade e mesmo para a sobrevivência das empresas (RASERA; BALBINOT, 2010), pois o ambiente econômico é caracterizado pela competição global (JOHNSON; SCHOLES; WHITTINGTON, 2007; GARRETÓN et al. 2007; GRÖNLUND; SJÖDIN; FRISHAMMAR, 2010). Essa crescente competitividade faz com que as organizações busquem resultados mais expressivos, adotando estratégias que vão desde inovações em produtos, serviços e processos até a formação de parcerias com clientes, concorrentes e fornecedores, com o objetivo de fortalecer as relações e obter melhores condições de negociação e manutenção de mercado. Nisiyama e Oyadomari (p.189, 2012) relatam que "as empresas têm revisto suas estratégias buscando formas alternativas de inovação, terceirizando seu processo de pesquisa, através de aquisições, alianças ou apenas buscando formas de cooperação".

A inovação é um fator que contribui para a competitividade empresarial, sendo considerada por Abulrub e Lee (2012) como o ponto principal para manter uma vantagem competitiva em um mercado e ganhar a liderança. A capacidade de inovar, no entanto, depende de muitos fatores para que seja alcançada com sucesso, dentre eles, destacam-se: o clima organizacional, a cultura, os processos internos e o ambiente externo.

Por sua vez, a inovação em pequenas e médias empresas (PME) é prejudicada pela falta de recursos financeiros. As oportunidades são escassas para recrutar trabalhadores e para pequenas inovações (VAN DE VRANDE et al. 2009; GASSMANN; ENKEL; CHESBROUGH, 2010; CREMA; VERBANO; VENTURINI, 2014; COLOMBO; PIVA; ROSSI-LAMASTRA, 2014). Assim sendo, a inovação aberta (IA) se torna ainda mais importante para empreendimentos desse porte, pois torna possível acessar recursos e capacidades que estão em outros atores. O modelo de inovação aberta propõe às organizações o uso de conhecimento e ideias internas e externas para o desenvolvimento de inovações. Utiliza caminhos internos e externos para explorar essas mesmas inovações, sem a necessidade de as empresas construírem sozinhas decisões completas para disponibilizá-las ao mercado (CHESBROUGH, 2004).

A utilização da cooperação permite às organizações reduzir custos com pesquisa e desenvolvimento (P\&D) (TIDD; BESSANT; PAVITT, 2001; CHESBROUGH; VANHAVERBEKE; WEST, 2006; TSAI; WANG, 2009) e possibilita ficarem atentas às oportunidades que o mercado pode oferecer (CHESBROUGH, 2012). Além disso, a empresa pode ampliar as suas oportunidades na maximização dos ganhos com P\&D. Assim, colaboram com outras empresas no desenvolvimento e fabricação de novos produtos. Desse modo, a IA é vista como uma forma de enfrentar as pressões de ambientes competitivos, os ciclos de vida curtos e os produtos de alta tecnologia (DITTRICH; DUYSTERS, 2007).

A opção pelo foco da pesquisa em PME deve-se ao reduzido número de estudos que analisa a IA em empreendimentos desse porte (VAN DE VRANDE et al. 2009; LEE et al. 2010; WYNARCZYK; PIPEROPOULOS; MCADAM, 2013). Grande parte das pesquisas em IA é direcionada às empresas de alta tecnologia e às empresas multinacionais (SILVA; DACORSO, 2013; VAN DE VRANDE et al. 2009; WESTERGREN; HOLMSTRÖM, 2012; WYNARCZYK; PIPEROPOULOS; MCADAM, 2013). Adicionalmente, as PME são excluídas da corrente principal de estudos sobre a IA. Os pesquisadores ainda não exploraram em profundidade como as PME podem fazer uso de laços externos para criarem fontes de ideias e conhecimento para a inovação (BRUNSWICKER; VANHAVERBEKE, 2014), e inovar é um desafio para empresas desse porte, pois elas possuem mais limitações e dificuldades de recursos financeiros e capacidades do que grandes empresas. Dessa forma, a IA torna-se uma alternativa estratégica para as empresas desse porte, pois podem adotar práticas de IA para promover a geração de inovações. As práticas de IA tornam-se altamente relevantes para as PME frente às limitações de recursos e de ativos tecnológicos (NARULA, 2004). 
Diante desses fatos, este ensaio teórico tem por objetivo identificar como a IA foi relacionada à vantagem competitiva para as PME nos principais estudos existentes, como também prover uma agenda para futuras pesquisas. O método utilizado foi o da pesquisa bibliográfica. Os artigos foram identificados através de uma pesquisa nas bases de dados Web of Science utilizando-se a frase "open innovation" no título, resumo e palavras-chave. Os artigos, posteriormente, foram selecionados com base em seus conteúdos (primeiramente, resumo, depois no texto completo), a fim de eliminar os artigos que retornaram na busca, mas não eram relacionados ao objetivo deste trabalho.

Este artigo está estruturado em seis seções, além da introdução. São elas: "Vantagem competitiva nas organizações", em que são abordados os principais conceitos; "As PME no ambiente dos negócios", em que são apresentadas suas características, peculiaridades e os desafios enfrentados; "A inovação aberta", em que são apresentadas algumas práticas relacionadas a esse modelo; "a inovação aberta como vantagem competitiva para as PME, abordando os achados dos principais trabalhos empíricos encontrados na literatura", em que se demonstra de que forma esse modelo pode gerar valor e servir como uma alternativa de desenvolvimento e crescimento. A seção 3 discute os achados à luz da literatura e oferece sugestões para futuras pesquisas. Por fim, nas considerações finais, são indicadas as contribuições deste artigo.

\section{Referencial Teórico}

Esta seção discute os estudos sobre vantagem competitiva e IA em PME.

\subsection{Vantagem competitiva nas organizações}

Este é um tema bastante discutido na literatura em torno dos diferenciais que alavancam um aumento de fatias de mercado, pois algumas empresas conseguem alcançar, enquanto outras lutam para adquirir a vantagem competitiva. O termo foi concebido por Michael Porter (1989) em seu livro "Vantagem competitiva", em que traz sua definição, que é a criação de valor para as organizações por meio de estratégias bemsucedidas. Assim, permite um alto desempenho frente ao mercado e aos concorrentes. Barney (1991) considera vantagem competitiva quando uma empresa executa uma estratégia de criação de valor que não pode ser executada por outro concorrente, assim gerando mais valor econômico do que seus concorrentes.

A magnitude da vantagem competitiva de uma empresa é em função do valor dos seus recursos e capacidades, ou seja, as empresas cujos recursos e as capacidades são de valor marginal, na melhor das hipóteses, irão alcançar uma vantagem competitiva pequena. Por outro lado, as empresas cujos recursos e capacidades são de grande valor provavelmente irão alcançar vantagens competitivas consideráveis (RUMELT, 1991; NEWBERT, 2008). Brandenburger e Stuart Jr. (1996) descrevem a vantagem competitiva considerando a criação de valor entre a intenção a pagar pelo cliente e o custo de oportunidade dos fornecedores.

A vantagem competitiva é um conceito-chave na gestão estratégica (WIGGINS; RUEFLI, 2002). Também é observada através de um desempenho superior (POWELL, 2001) sendo uma possibilidade que a empresa tem de se destacar e dominar o mercado (BRITO e BRITO 2012). Woerter e Roper (2010) mencionam que, devido às incertezas ambientais e mudanças quanto ao futuro, a busca por caminhos de atuação tem se tornado mais que um propósito central nas empresas. A vantagem competitiva torna-se uma necessidade de sobrevivência. Além disso, por causa das preferências dos clientes inconstantes e as tecnologias em evolução, a vantagem competitiva da empresa habita nas competências que permitem acesso da empresa a uma grande variedade de mercados (CARNEIRO; CAVALCANTI; SILVA, 1997).

A empresa ganha vantagem competitiva quando executa atividades estrategicamente importantes e de uma forma mais barata ou melhor do que a concorrência (PORTER, 1989). Esta é a forma, através de iniciativas de inovação (PORTER, 1999). A inovação é um mecanismo fundamental através do qual as empresas garantem os lucros competitivos (VAN DE VEN, 1986). As empresas devem incorporar suas competências em produtos e serviços e criar transações com clientes para levar uma vantagem competitiva (MCGRATH, et al. 1996).

Por sua vez, a vantagem competitiva sustentada consiste em estratégias que criam valor e diferencial para a empresa e que não possuem benefícios replicados por nenhum concorrente (ADNER; ZEMSKY, 
2006). Barney (1991) descreve que as fontes de vantagem competitiva sustentada de uma empresa se encontram em sua heterogeneidade e na imobilidade dos recursos da empresa, os quais são: a) recursos valiosos: recursos que permitem que as estratégias implementadas sejam aprimoradas para garantir eficiência e eficácia; b) recursos raros: recursos que criam valor para a empresa e não são implementados por um grande número de empresas; c) recursos imperfeitamente imitáveis (inimitáveis): recursos adquiridos e explorados pela empresa como diferenciais em função das suas condições históricas, da sua ambiguidade casual e da sua complexidade social; d) substituibilidade: recursos que podem ser substituídos por recursos semelhantes sem afetar as estratégias ou recursos que são substituídos estrategicamente para atingir a metas propostas.

Na construção do conceito de vantagem competitiva, sob o aspecto de percepção do valor, pode-se entender que a vantagem competitiva é o excedente, além do custo que o cliente está disposto a pagar (BESANKO et al., 2007). Para que ocorra agregação de valor, é necessário um aumento de investimentos em ativos específicos, porém é importante alertar sobre custos de transação, e, nesse aspecto, estudos de Dyer (1997), Williamson (1981) e Mcivor (2009) mostraram que o investimento em ativos específicos aumenta os custos de transação. O valor percebido pelo cliente é construído a partir da utilização da capacidade de inovação das organizações, tendo como base os ativos (ADNER; ZEMSKY, 2006; MORROW et al., 2007). Conforme Porter (1989), há dois posicionamentos de valor que conferem vantagens competitivas: uma vantagem de custo ou uma vantagem de diferenciação. Finalmente, a partilha de atividades de valor irá resultar em uma vantagem competitiva desde que essa atividade apresente uma fração de custos operacionais e que possam ser reduzidos através do compartilhamento (PORTER, 1989), que nos remete ao conceito de inovação aberta, discutido adiante.

\subsection{PME no ambiente dos negócios}

A definição de PME varia de acordo com a estrutura ou tamanho de mercado de cada país. No Brasil, as definições da microempresa e empresa de pequeno porte (MPE), dadas pela Lei n. ${ }^{\circ}$ 9.841/99 e pela Lei do Simples, n. ${ }^{\circ}$ 9.317/96, utilizam o critério da receita bruta anual. Já os critérios utilizados pela Relação Anual de Informações Sociais (RAIS) e o Ministério do Trabalho e Emprego (MTE), juntamente com o Sebrae, caracterizam o porte da empresa através do número de empregados. O Sebrae (2004) classifica como de pequeno porte as empresas brasileiras na faixa de 20 a 99 empregados (para indústrias) e 10 a 49 empregados (para comércio e serviços). Já as médias empresas, de 100 a 499 empregados (para indústria) e 50 a 99 empregados (para comércio e serviços).

As PME representam 5,7 milhões de empresas no Brasil (SEBRAE, 2012). Destas, 99\% são consideradas micro e pequenas empresas. Além disso, de acordo com o Sebrae (2012) e o Instituto Brasileiro de Geografia e Estatística (IBGE, 2006), esse porte de empresas gera a maior parcela de emprego e renda no Brasil. As PME são consideradas importantes para a economia, por sua capacidade de gerar empregos e contribuírem para a economia. São importantes para manter o forte crescimento econômico (ATES et al., 2013). Cândido e Abreu (2000) consideram essas empresas como o motor do desenvolvimento econômico em uma sociedade. No entanto, elas têm dificuldade na atuação por serem de pequeno porte e por sofrerem pressões vindas do mercado e internamente. Lee et al. (2010) fazem alguns destaques com relação às dificuldades encontradas por empresas desse porte, conforme apresenta o Quadro 1:

Quadro1: Dificuldades encontradas em PME

\begin{tabular}{|l|l|}
\hline \multicolumn{1}{|c|}{ Ambiente interno da empresa } & \multicolumn{1}{c|}{ Ambiente externo da empresa } \\
\hline Falta de empregados com competência para o & Dificuldade para obter crédito, devido ao elevado \\
negócio; & risco de incerteza tecnológica; \\
Pouco conhecimento de mercado; & Dificuldade para encontrar pessoas qualificadas; \\
Pouca informação tecnológica; & Incertezas de mercado no que se refere à criação de \\
Altos custos relacionados à comercialização de & novos produtos. \\
produtos para inovação; & \\
Pouca capacidade na gestão de P\&D. & \\
\hline
\end{tabular}

Fonte: Elaborado pelos autores a partir de Lee et al. (2010). 
Barnett e Storey (2000) destacam que os negócios de pequeno e médio porte enfrentam desafios em se tratando da inovação. As PME possuem várias limitações, como a falta de recursos para P\&D, processos de inovação não estruturados e capacidades internas subdesenvolvidas (PARIDA; WESTERBERG; FRISHAMMAR, 2012). Outro desafio que enfrentam são as limitações de fontes de informação, o que torna difícil para os clientes potenciais avaliarem se pode ou não ser lucrativo fazer negócio com a empresa (CHOLLET; GÉRAUDEL; MOTHE, 2014). Elas também carecem da capacidade em fabricação, distribuição e comercialização, que são indispensáveis para introduzir inovações de uma forma eficaz no mercado (NARULA, 2004). Para esse porte de empresas, torna-se mais difícil controlar todas as partes no processo de inovação, visto que são necessários diferentes tipos de recursos e competências. Elas geralmente são mais flexíveis, menos formalizadas e mais ágeis na tomada de decisões, porém os recursos financeiros para P\&D são limitados (VOSSEN, 1998; VAN DE VRANDE et al., 2009).

Historicamente, as PME apresentam enormes dificuldades no mundo concorrencial, pois elas possuem desconhecimento de mercado, necessidade de treinamento de mão de obra e de atualizações de tecnologias e, principalmente, pouco uso de instrumentos de gestão, fatores que dificultam as iniciativas pelos gestores. A capacidade de inovação é uma das dinâmicas mais importantes, pois permite a PME obterem um elevado nível de competitividade. Assim, a forma de promover e sustentar uma capacidade de inovação deve ser 0 foco principal dos gestores em PME (ÇAKAR; ERTÜRK, 2010).

Devido a sua pequena dimensão e a limitações de recursos, as PME não podem cobrir todas as atividades de inovação necessárias para obter sucesso na inovação (BRUNSWICKER; VANHAVERBEKE, 2014). Diante dos desafios enfrentados, as PME estão optando às redes para melhorar a sua capacidade em inovar (GARDET; FRAIHA, 2012). Muitas dessas empresas procuram associar-se às redes para enfrentar a concorrência com as grandes empresas. Assim, elas desenvolvem inovações com o auxílio de outros atores, como fornecedores, usuários, instituições de pesquisa e, até mesmo, concorrentes. Dessa forma, a IA torna-se uma alternativa estratégica para que empresas desse porte consigam manter-se no mercado.

Em alguns estudos, como Brunswicker e Vanhaverbeke (2014), demonstrou-se que as PME se envolvem na busca do conhecimento externo para que possam garantir sua fonte de inovação. Konsti-Laakso, Pihkala e Kraus (2012) exploraram que a forma de inovar em PME pode ser facilitada através do desenvolvimento das redes de inovação. A participação através dessas redes oferece uma solução para empresas desse porte, visto que elas têm menos recursos e capacidades para inovar.

\subsection{Inovação aberta}

No modelo de inovação, as empresas apoiam-se na P\&D, que acontecem em seus laboratórios internos, detendo uma vantagem competitiva sustentada (CHESBROUGH, 2003; GANN, 2004). No entanto muitos projetos ficavam engavetados, os que não são complementares ao negócio principal da empresa (GANN, 2004; BLAU, 2007).

Além disso, no processo de inovação fechada não há uma relação com o ambiente externo para interações ou na troca de opiniões (CHESBROUGH, 2003). Esse modelo é caracterizado por poucas fontes de inovação; tem-se um número limitado de fontes externas ou somente fontes internas (PADILLAMELÉNDEZ; AGUILA-OBRA; LOCKETT, 2012; SENER; HOBIKUGLU, 2013). Eventualmente, tem-se a busca por algum conhecimento específico em universidade ou em algum instituto de pesquisa, mas tendo sempre cuidado com o sigilo das informações (STAL; NOHARA; CHAGAS Jr., 2014). Dessa forma, todo trabalho de criação é feito pelo setor interno responsável da empresa, ou seja, através do setor de P\&D (SENER; HOBIKOGLU, 2013).

Chesbrough e Crowther (2006) explicam que as empresas investem em grandes departamentos para coordenar a inovação e proporcionar um crescimento sustentável. Porém, esse modelo está erodindo, devido a alguns fatores (CHESBROUGH; CROWTHER, 2006). Sendo assim, Chesbrough (2003) observou o comportamento histórico das grandes empresas americanas ao longo do século XX. O modelo de gestão da inovação nessas empresas foi bastante fechado, no que diz respeito ao surgimento de ideias e sua aplicação no mercado. Esse modelo baseava-se em autossuficiências, as ideias somente eram desenvolvidas internamente (VANHAVERBEKE; VAN DE VRANDE; CHESBROUGH, 2008). 
Sendo assim, no modelo de inovação fechada, as empresas geram, desenvolvem e comercializam suas próprias ideias (CHESBROUGH, 2003). Chesbrough (2003), no entanto, destacou alguns fatores que promoveram mudanças no mercado concorrencial, afetando a estratégia de inovação das empresas, como a crescente mobilidade da mão de obra, o surgimento de centros de formação de excelência em todo o mundo, a perda de hegemonia dos EUA, Europa e Japão para outras regiões emergentes e o elevado investimento em capital empreendedor. Desde então, ficou cada vez mais difícil para as empresas controlarem todos os recursos e competências necessárias para inovar.

A inovação passou por uma mudança fundamental na forma de como é gerada e levada ao mercado. Surgiu um novo paradigma de inovação, denominada por Chesbrough (2003) de IA. A IA passou a ser uma estratégia fundamental para as organizações, contribuindo para gerar vantagem competitiva (CHESBROUGH; CROWTHER, 2006; NOHARA; CHAGAS Jr., 2014). Esse modelo parte do pressuposto que o conhecimento é amplamente disponível, as pesquisas estão ficando cada vez mais caras e o ciclo de vida dos produtos está diminuindo (VAN DE VRANDE et al. 2009). Há um crescente aumento na velocidade de lançamento de novos produtos, o que torna os mercados cada vez mais dinâmicos, portanto, torna-se mais difícil para uma organização inovar de forma solitária.

Uma organização que adota o modelo de IA aumenta a oportunidade de gerar valor (CHESBROUGH; APPLEYARD, 2007). Além do mais, desenvolvem suas inovações com o auxílio de outros atores, como fornecedores, usuários, instituições de pesquisa e, até mesmo, concorrentes. Muitas empresas inovadoras mudaram a forma na busca de novas ideias, adotando estratégias de busca abertas, que envolvem o uso de uma ampla gama de atores externos como fontes para ajudá-los a alcançar e sustentar a inovação (LAURSEN; SALTER, 2006). A abertura de fontes externas permite às empresas atrair ideias de pessoas de fora para aprofundar o conjunto de oportunidades disponíveis (LAURSEN; SALTER, 2006), envolvendo um processo de interação e de processamento de informações (LOVE; ROPER; VAHTER, 2014).

Algumas práticas de IA encontradas na literatura se referem à geração de ideias através da cadeia de valor. A colaboração com clientes proporciona às empresas um amplo conhecimento, a qual se faz necessário para inovações em produtos (UN; CUERVO-CAZURRA; ASAKAWA, 2010). Usuários de produtos são as fontes de conhecimento externo mais importante de uma organização (CHATTERJI; FABRIZIO, 2014). A colaboração com esse ator proporciona à empresa um amplo conhecimento das necessidades, as quais são necessárias para inovar. As colaborações criativas com os usuários aumentam a inovação de produtos corporativos e os benefícios são maiores em áreas de novas tecnologias e na geração de inovações radicais (CHATTERJI; FABRIZIO, 2014). Os funcionários estão entre os mais importantes tipos de geração de recursos, devido à escassez, especialização e conhecimento tácito (BELENZON; SCHANKERMAN, 2014).

Outra prática são as parcerias de codesenvolvimento. Essa prática consiste em conduzir ou participar de projetos com parceiros externos garantindo a qualidade (PITASSI, 2012). Essas parcerias objetivam uma relação de trabalho entre duas ou mais partes na criação e entrega de um novo produto, tecnologia ou serviço.

Outra prática é a geração de receita a partir de P\&D interno, que permite gerar receitas através da venda e licenciamento de tecnologias, ou mesmo pela geração de empresas spin-offs. O surgimento de novas empresas a partir do conhecimento interno, o que implica na geração de spin-off e processos de spinout. A base da organização-pai também poderá abranger finanças, recursos humanos, assessoria jurídica e serviços administrativos, entre outros (VAN DE VRANDE et al. 2009).

A cooperação entre empresas e o sistema científico e tecnológico proporciona a geração de conhecimento que, dificilmente, seriam gerados de forma individualizada. As colaborações com universidades têm um forte impacto em inovações, pois elas possuem um conhecimento muito amplo e as barreiras de acesso a elas são reduzidos (COHEN; LEVINTHAL, 1990; UN; CUERVO-CAZURRA; ASAKAWA, 2010).

As empresas podem terceirizar suas atividades de P\&D para adquirir o conhecimento externo (VAN DE VRANDE et al. 2009). Essa prática oferece uma redução de custos e riscos às empresas e permite utilizar ativos complementares para impulsionar o crescimento (GASSMANN; ENKEL; CHESBROUGH, 2010).

Grandes empresas que adotam práticas IA já foram estudadas na literatura, como Dutch State Mine (DSM) (KIRSCHBAUM, 2005) e Italcementi (CHIARONI; CHIESA; FRATTINI, 2011). A Procter \& Gamble (P\&G) é um caso exemplar de empresa que integrou práticas de estímulo a IA (DODGSON; GANN; SALTER, 2006). 
Após adotar o modelo de negócios abertos, desenvolveu um programa chamado Conectar e desenvolver, que licencia produtos de outras empresas e os leva ao mercado como marcas da P\&G (CHESBROUGH, 2012). Dessa forma, eles anunciaram que aumentaram suas vendas em uma taxa de $50 \%$ e a eficiência do seu P\&D em 60\% após adotar esse modelo (ENKEL; GASSMANN; CHESBROUGH, 2009).

\subsection{Inovação aberta uma vantagem competitiva para PME}

A IA oferece uma perspectiva de custos mais baixos para inovação, tempo mais curto para entrar no mercado e a chance de reduzir os riscos com as outras empresas (CHESBROUGH; 2012; STAL; NOHARA; CHAGAS Jr., 2014). Chesbrough, Vanhaverbeke e West (2006) destacam que as parcerias nas atividades de P\&D são um recurso para as capacidades internas da empresa. Em complemento, Tidd, Bessant e Pavitt (2001) e Chesbrough, Vanhaverbeke e West (2006) apontaram que explorar recursos de outras empresas, externas à empresa, consequentemente reduz o risco de entrada no mercado e o tempo de desenvolvimento de um novo produto.

Nesse modelo de negócios, as chances de erro referentes ao desenvolvimento do produto ou serviço são minimizadas, pois, como existem muitos atores envolvidos, isso faz com que haja uma maior visão sobre um determinado produto ou serviço (CHESBROUGH; VANHAVERBEKE; WEST, 2006), podendo até algumas ideias serem vendidas caso não atendam às necessidades da empresa. Esse modelo de negócios oferece a capacidade para geração de novas ideias e de realizar pesquisas com menores custos e riscos, como também a possibilidade de ampliação do negócio (CHESBROUGH; VANHAVERBEKE; WEST, 2006). Assim, as empresas têm maior liberdade para investir e, consequentemente, uma redução nos riscos investidos na inovação (CHESBROUGH, 2003, 2006;TSAI; WANG, 2009), além de o desenvolvimento tecnológico ser mais rápido (ERZURUMLU, 2010).

Chesbrough, Vanhaverbeke e West (2006) indicaram que, nesse modelo de negócio de IA, há um aumento nas possibilidades de incremento nas receitas com a comercialização da propriedade intelectual (PI), criação de spin-offs e joint-ventures, como novas formas de negócio. Sendo assim, as PME podem buscar ajuda em universidades, institutos de pesquisa, clientes, colaboradores, fornecedores, dentre outros citados anteriormente, para o desenvolvimento de inovações, sem ter propriamente que contratar o melhor profissional do mercado, podendo também lucrar caso desenvolva alguma propriedade intelectual e não faça diretamente a comercialização, pois os direitos comerciais dessa PI poderão ser vendidos a outras empresas. As chances de erros são minimizadas, pois elas têm maior liberdade para investir e o risco é compartilhado com os demais atores.

A utilização da cooperação permite às empresas a redução de custos com P\&D (TIDD; BESSANT; PAVITT, 2001; CHESBROUGH; VANHAVERBEKE; WEST, 2006; TSAI; WANG, 2009) e possibilita, ainda, ficarem atentas ao que o mercado pode oferecer (CHESBROUGH, 2012a). Há ampliação das oportunidades para a empresa na maximização dos ganhos com P\&D (CHESBROUGH; VANHAVERBEKE; WEST, 2006), podendo gerar maior capacidade de realizar pesquisas estratégicas com baixos níveis de riscos, com acesso à tecnologia e a conhecimentos. Dessa forma, elas elevam o seu poder de competitividade, pois produzem relações mais confiantes com seus fornecedores e clientes, concorrendo de forma mais equilibrada com as grandes empresas (OLIVEIRA; GUERRINI, 2002).

Com o intuito de facilitar a compreensão, o Quadro 2 apresenta uma síntese dessa seção.

Quadro 2: Síntese das vantagens da inovação aberta

\begin{tabular}{|c|c|c|c|c|}
\hline Estudo & Tipo de estudo & Aspecto abordado & Ideias centrais & $\begin{array}{c}\text { Implicações para os objetivos } \\
\text { do estudo }\end{array}$ \\
\hline $\begin{array}{c}\text { Stal, Nohara e Chagas } \\
\text { Jr. (2014); } \\
\begin{array}{c}\text { Chesbrough (2012) } \\
\text { Silva e Zilber (2013) }\end{array}\end{array}$ & $\begin{array}{c}\text { Empírico (3 } \\
\text { empresas) } \\
\text { Teórico } \\
\text { Empírico (3 } \\
\text { empresas) }\end{array}$ & $\begin{array}{c}\text { Perspectiva de baixo } \\
\text { custo para inovar. }\end{array}$ & $\begin{array}{c}\text { Os custos são divididos } \\
\text { entre os demais atores. }\end{array}$ & $\begin{array}{c}\text { Os baixos custos se tornam } \\
\text { indispensáveis para essas } \\
\text { empresas, devido ao cenário } \\
\text { competitivo e mudanças nos ciclos } \\
\text { de vida dos produtos. }\end{array}$ \\
\hline
\end{tabular}


Quadro 2: Síntese das vantagens da inovação aberta. (continuação)

\begin{tabular}{|c|c|c|c|c|}
\hline $\begin{array}{c}\text { Grimaldi, Quinto e Rippa } \\
\text { (2013) } \\
\text { Chesbrough (2012) } \\
\text { Tidd, Bessant e Pavitt } \\
\text { (2001) }\end{array}$ & $\begin{array}{c}\text { Empírico (4 } \\
\text { empresas) } \\
\text { Teórico } \\
\text { Teórico }\end{array}$ & $\begin{array}{c}\text { Redução de riscos } \\
\text { de entrada no } \\
\text { mercado e tempo de } \\
\text { desenvolvimento de um } \\
\text { novo produto. }\end{array}$ & \begin{tabular}{|c|} 
Exploram recursos \\
e tecnologias com \\
outras empresas \\
externas e os riscos são \\
compartilhados pelos \\
demais atores. \\
\end{tabular} & $\begin{array}{l}\text { O mercado encontra-se altamente } \\
\text { competitivo, dificultando a entrada } \\
\text { de novas empresas, desta forma } \\
\text { os riscos são compartilhados pelos } \\
\text { demais atores através da nova } \\
\text { estratégia de negócios, a IA. }\end{array}$ \\
\hline $\begin{array}{c}\text { Chesbrough, } \\
\text { Vanhaverbeke e West } \\
(2006)\end{array}$ & Teórico & $\begin{array}{l}\text { Menores chances de } \\
\text { erro no desenvolvimento } \\
\text { de um novo produto/ } \\
\text { serviço. }\end{array}$ & $\begin{array}{c}\text { É compartilhado pelos } \\
\text { demais atores. }\end{array}$ & $\begin{array}{l}\text { A interação entre os diversos } \\
\text { atores oferece uma ampla } \\
\text { visão do produto/serviço, desta } \\
\text { forma minimizando erros no } \\
\text { desenvolvimento. }\end{array}$ \\
\hline $\begin{array}{l}\text { Parida, Westerberg e } \\
\text { Frishammar (2012) } \\
\text { Chesbrough, } \\
\text { Vanhaverbeke e West } \\
\text { (2006) }\end{array}$ & $\begin{array}{c}\text { Empírico (252 } \\
\text { empresas) } \\
\text { Teórico }\end{array}$ & $\begin{array}{c}\text { Capacidade para } \\
\text { geração de novas ideias } \\
\text { e de realizar pesquisas } \\
\text { com menores custos e } \\
\text { riscos. }\end{array}$ & $\begin{array}{c}\text { O conhecimento } \\
\text { é compartilhado } \\
\text { pelos demais atores } \\
\text { no processo. E } \\
\text { as pesquisas são } \\
\text { realizadas em parcerias } \\
\text { internas e externas à } \\
\text { empresa. }\end{array}$ & $\begin{array}{l}\text { Conhecimentos e ideias são } \\
\text { gerados pelos colaboradores em } \\
\text { iniciativas de inovação e vínculos } \\
\text { informais entre colaboradores e } \\
\text { entre empresas. As empresas } \\
\text { muitas vezes não possuem } \\
\text { todos os recursos e capacidades } \\
\text { necessárias para inovar. Esse } \\
\text { modelo torna-se uma forma de } \\
\text { suprir essas faltas. }\end{array}$ \\
\hline $\begin{array}{c}\text { Spithoven, } \\
\text { Vanhaverbeke e } \\
\text { Roijakkers (2013) } \\
\text { Brunswicker e } \\
\text { Vanhaverbeke (2014) } \\
\text { Chesbrough, } \\
\text { Vanhaverbeke e West } \\
\text { (2006) }\end{array}$ & $\begin{array}{c}\text { Empírico (1427 } \\
\text { empresas) } \\
\text { Empírico (1411 } \\
\text { empresas) } \\
\text { Teórico }\end{array}$ & $\begin{array}{l}\text { Aumento nas receitas da } \\
\text { empresa. }\end{array}$ & $\begin{array}{l}\text { Ocorre através da } \\
\text { comercialização da PI, } \\
\text { criação de empresas } \\
\text { spin-offs e joint } \\
\text { ventures. }\end{array}$ & $\begin{array}{c}\text { A empresa poderá lucrar caso } \\
\text { desenvolva alguma tecnologia e } \\
\text { não comercialize o produto final, } \\
\text { ao vender a tecnologia a outras } \\
\text { empresas. }\end{array}$ \\
\hline
\end{tabular}

Fonte: Elaborado pelos autores.

Esse modelo aberto de inovar representa uma alternativa competitiva para o desenvolvimento de PME, ajudando-as a superar um pouco as dificuldades que encontram no ambiente interno e externo à empresa, conforme anteriormente disposto. Mesmo que tais problemas não possam ser completamente superados, vários exemplos evidenciam que seus efeitos podem ser amenizados pelo novo modelo de inovação, ou seja, através de uma forma mais aberta de inovar, através da formação de redes e parcerias.

Dessa forma, elas podem explorar os recursos e tecnologias com outras empresas, reduzindo o risco de entrada no mercado, minimizando os erros no desenvolvimento de um produto/serviço, tendo maior facilidade para conseguir algum financiamento, além de ficarem mais próximas das necessidades dos clientes/consumidores. A competitividade se encontra nas relações que estabelece com a sociedade. Conforme descrevem Robertson, Casali, Jacobson (2012), a absorção do conhecimento através do modelo de IA favorece essas empresas, pois elas capturam, através de fontes externas, conhecimento e aprendizado que se tornam indispensáveis para competir no mercado. Em complemento, Mariano, Guerrini e Rebelatto (2012) afirmam que cada vez mais as empresas têm percebido as vantagens de estabelecer alianças com outras empresas, formando redes. As vantagens relacionadas a esse fato referem-se aos indicadores de desempenho.

\section{Discussão da literatura existente e sugestões para novas pesquisas}

O estudo teve como objetivo fazer uma revisão de literatura demonstrando como a IA pode gerar vantagem competitiva para as PME. Dessa forma, o estudo contribui para pesquisadores da área e executivos que buscam inovações. Gestores poderão aplicar as práticas de IA em suas empresas, promovendo inovações. A inovação constitui um dos fatores mais importantes para incrementar a competitividade das empresas. Em especial as PME, consideradas importantes para a economia por sua capacidade de gerar empregos e contribuir para a economia local. Sendo assim, no ponto de vista social, o estudo pode contribuir para a sobrevivência e competitividade das PME. Desse modo, a IA em PME contribui: favorecendo a economia local; promovendo o crescimento econômico; garantindo a sobrevivência, renda e emprego; melhorando as 
condições de vida da população; bem como contribuindo para que essas empresas sobrevivam por mais tempo. De certa maneira, esse trabalho dá continuidade e aprofunda os achados de Silva e Dacorso (2013) ao oferecer uma consolidação dos achados empíricos (ver Quadro 2) e propor novas avenidas de pesquisa.

Assim, como contribuições deste estudo, podem ser apontadas os achados deste artigo para a literatura, sobretudo no que diz respeito à criação e apropriação de valor. Os autores Brandenburger e Stuart Jr. (1996) explicam que a criação de valor depende das características de três categorias de jogadores: cadeia de fornecimento, empresas e compradores. No entanto surgem algumas questões, como: quanto valor um jogador poderá conquistar?

Brito e Brito $(p .71,2012)$ descrevem que o "valor criado não é necessariamente o valor apropriado; enquanto o custo de oportunidade e disposição a pagar define o valor criado, preço e custo definem valor apropriado". Da mesma forma, quais critérios ou o que irá determinar o quanto de valor cada jogador poderá se apropriar? Quem fica com a maior parte desse valor apropriado? Quais as vantagens e desvantagens dessas questões para as PME no processo da IA? Daí decorre nossa primeira sugestão de pesquisa, que é identificar os fatores preditores da apropriação do valor gerado nas IAs, especialmente aquelas que envolvam PME. Por exemplo: o valor é apropriado igualmente entre as empresas em uma IA ou a apropriação de valor é proporcional ao porte da empresa?

Outra questão em aberto na literatura é o impacto social e ambiental das inovações abertas. Perguntas de pesquisa nesse tema seriam, por exemplo: inovações abertas envolvendo PME enfatizam mais inovações sociais do que as ambientais? As inovações abertas de PMEe empresas de grande porte são mais propícias (ou menos) do que as inovações abertas em que apenas PME participam?

Finalmente, outro ponto a refletir - embora de natureza mais metodológica do que teórica - é a questão da coleta dos dados de inovação aberta. Os estudos tradicionais de inovação tecnológica geralmente se baseiam em estudos de patentes e nos gastos em P\&D. Entretanto capturar inovações abertas, mesmo em grandes empresas, é difícil. Essa tarefa, em se tratando de PME, é ainda mais desafiadora. O uso de levantamentos tipo survey, comuns na literatura, limitam uma análise mais longitudinal do fenômeno. Portanto, todas as inferências de causa e efeito (por exemplo, relacionando IA e desempenho) são questionáveis do ponto de vista da endogenia. Dessa forma, por exemplo, não há como saber se uma observação de correlação entre IA e desempenho se deve: ao fato de IA levar a um melhor desempenho, ao fato das PME com melhor desempenho terem mais condições de investir em IAs ou, ainda, uma terceira causa (por exemplo, em longo prazo), se ao fato de influenciar ambas as variáveis (IA e desempenho) nas PME.

\section{Considerações finais}

As mudanças na forma de competir das empresas desencadearam a crescente necessidade de inovação. $O$ aumento da concorrência e a formação de consumidores cada vez mais exigentes à satisfação aumentaram os investimentos em tecnologia como fonte de criação de valor, tornando dispendioso o processo de renovação das organizações.

Portanto, as organizações precisam ter visão de estratégia, olhando para si mesmas e modelando a estratégia de modo a permitir uma melhor exploração de seus recursos internos e competências. Os gestores precisam interpretar seu ambiente externo. As empresas devem olhar para seu interior com o objetivo de alavancar os recursos que lhes proporcionem uma vantagem em relação aos seus concorrentes e implementar uma estratégia em consonância com suas capacidades.

O modelo de inovação aberta é uma alternativa de estratégia para as pequenas e médias empresas, podendo ser considerada uma vantagem competitiva para o negócio. A inovação aberta torna-se um modelo especialmente atraente para pequenas e médias empresas, pois os recursos financeiros e capacidades são mais escassos nos investimentos em P\&D interno. Assim, as parcerias externas podem representar uma forma mais eficiente de desenvolvimento de inovações. As práticas de inovação aberta demonstraram, nos estudos encontrados, ter efeitos positivos e proporcionar lucros para a empresa. Tais práticas incluem: a geração de ideias através da cadeia de valor; parcerias de codesenvolvimento; geração de receitas a partir de P\&D interno (comercialização de tecnologia); cooperação entre empresas e o sistema científico e tecnológico; e a terceirização das atividades de P\&D. 
O modelo aberto pode proporcionar às PME mais lucro, pois elas aproveitam as tecnologias e conhecimentos desenvolvidos por outras empresas e partilham através de parcerias e alianças para desenvolver as inovações. Outra vantagem para essas empresas está na captação de recursos entre as empresas a um baixo custo, pois elas abordam obstáculos, como a localização e os recursos financeiros, humanos e tecnológicos internos, que dificultam o desenvolvimento de novos produtos e o acesso a novos mercados.

Processos de inovação exigem que as empresas dominem conhecimentos altamente específicos sobre os diferentes usuários, tecnologias e mercados. Portanto, é importante a formação de parcerias com os agentes externos à organização, pois eles proporcionam um pensamento aberto, necessário e indispensável para aproveitar os fluxos de informações.

Assim, o modelo de negócios abertos oferece uma perspectiva positiva para as empresas desse porte, mostrando-se uma alternativa de desenvolvimento que irá permitir associar valor e alcançar a vantagem competitiva. Gestores de pequenas empresas poderão aplicar o modelo de negócios aberto em suas organizações, promovendo inovações e mantendo-as competitivas no mercado. Esperamos, com este ensaio, oferecer aos gestores um portfólio de melhores práticas em inovação aberta e, à comunidade acadêmica, tanto uma revisão dos trabalhos empíricos quanto uma agenda de pesquisas futuras no tema.

\section{Referências}

ABULRUB, A. G; LEE, J. Open innovation management: challenges and prospects. Social and Behavioral Sciences, v. 41, p. 130-138, 2012.

ADNER, R.; ZEMSKY, P. A Demand-based perspective on sustainable competitive advantage. Strategic Management Journal, v. 27,p. 215-239, 2006.

ATES, A.; GARENGO, P.; COCCA, P.; BITITCI, U. The development of SME managerial practice for effective performance management. Journal of Small Business and Enterprise Development, v. 20, $n$. 1, p. 28-54, 2013.

BARNETT, E.; STOREY, J. Managers' accounts of innovation processes in small and medium-sized enterprises. Journal of Small Business and Enterprise Development,v. 7, n. 4, p. 315-324, 2000.

BARNEY, J. Firms resources and sustained competitive advantage. Journal of Management, v.17, n.1, p. 99-120, 1991.

BELENZON, S.; SCHANKERMAN, M. Motivation and sorting of human capital in open innovation. Strategic Management Journal, 2014.

BESSANT. J.; TIDD, J. Innovation and entrepreneurship; Chichester, West Sussex, England, John Wiley \& Sons, 2007.

BLAU, J. Philips Tears Down Eindhoven R\&D Fence. Research Technology Management, v.50, n. 6, p. 9-10, 2007.

BRANDENBURGER, A. M.; STUART, H. W. Jr. Value-Based Business Strategy. Journal of Economics \& Management Strategy, v. 5, n. 1, p. 5-24, 1996.

BRITO, R. P.; BRITO, L. A. L. Vantagem competitiva e sua relação com o desempenho: uma abordagem baseada em valor. Revista de Administração Contemporânea, v. 16, n.3, p. 360-380, 2012.

BRITO, R. P.; BRITO, L. A. L. Vantagem competitiva, criação de valor e seus efeitos sobre o desempenho. Revista de Administração de Empresa, v. 52, n. 1, p. 70-84, 2012.

BRUNSWICKER, S.; VANHAVERBEKE, W. Open innovation in small and medium-sized enterprises (SMEs): external knowledge sourcing strategies and internal organizational facilitators. Journal of Small Business Management, 2014.

ÇAKAR, N. D.; ERTÜRK, A. Comparing innovation capability of small and medium-sized enterprises: examining the effects of organizational culture and empowerment. Journal of Small Business Management, v. 48, n. 3, p. 325-359, 2010. 
CÂNDIDO, G. A.; ABREU, A. F. Aglomerados industriais de pequenas e médias empresas como mecanismo para promoção de desenvolvimento regional. Revista eletrônica de administração, v. $6, n$. 6, p. 1-26, 2000.

CARNEIRO, J. M. T; CAVALCANTI, M. A. F. D; SILVA, J. F. Porter Revisitado: análise crítica da tipologia estratégica do mestre. Revista de Administração Contemporânea, v.1, n.3, p. 7-30, 1997.

CHATTERJI, A. K.; FABRIZIO, K. R. Using users: when does external knowledge enhance corporate product innovation? Strategic Management Journal, v. 35, p. 1427-1445, 2014.

CHESBROUGH, H. W. Managing open innovation. in uncertain markets, new metrics can help companies to play poker as well as chess. Research- Tecnology Management, v. 47, n. 1, p. 23-26, 2004.

CHESBROUGH, H. W. Open Innovation: the new imperative for creating and profiting from technology. Harvard Business School Press, Boston, 2003.

CHESBROUGH, H. W. Modelos de negócios abertos: como prosperar no novo cenário da inovação. Porto Alegre: Bookman, 2012.

CHESBROUGH, H. W; APPLEYARD, M. M. Open Innovation and Strategy. California Management Review,v. 50, n. 1, p. 57-76, 2007.

CHESBROUGH, H. W; CROWTHER, A. K. Beyond high tech: early adopters of open innovation in other industries. R\&D Management v. 36, n. 3, p. 229-236, 2006.

CHESBROUGH, H.W; VANHAVERBEKE, W; WEST, J. Open Innovation: Researching a New Paradigm. OxfordUniversity Press, 2006.

CHIARONI, D.; CHIESA, V.; FRATTINI, F. The Open Innovation Journey: How firms dynamically implement the emerging innovation management paradigm. Technovation, v.31, n.1, p. 34-43, 2011.

CHOLLET, B.; GÉRAUDEL, M.; MOTHE, C. Generating business referrals for SMEs: the contingent value of CEOs' Social Capital. Journal of Small Business Management, v. 52, n. 1, p. 79-101, 2014.

COHEN, W. M, LEVINTHAL, D. A. Absorptive capacity: a new perspective of learning and innovation. Administrative Science Quarterly,v. 35, p. 128-152, 1990.

COLOMBO, M. G.; PIVA, E.; ROSSI-LAMASTRA, C. Open innovation and within-industry diversification in small and medium enterprises: the case of open source software firms. Research Policy, v. 43, n. 5, p. 891-902, 2014.

CREMA, M.; VERBANO, C.; VENTURINI, K. Linking strategy with open innovation and performance in SMEs". Measuring Business Excellence, v. 18, n. 2 p. 14-27, 2014.

DITTRICH, K.; DUYSTERS, G. networking as a means to strategy change: the case of open innovation in mobile telephony. Journal of Product Innovation Management, v. 24, n. 6, p. 510-521, 2007.

DODGSON, M.; GANN, D.; SALTER, A. The role of technology in the shift towards open innovation: the case of Procter \& Gamble. R\&D Management,v. 36, n. 3,p. 333-346, 2006.

DYER, J. H. Effective interfirm collaboration: how firms minimize transactions costs and maximize transaction value. Strategic Management Journal, v. 18, n. 7, p. 535-556, 1997.

ENKEL, E.; GASSMANN, O.; CHESBROUGH, H. Open R\&D and open innovation: exploring the phenomenon. R\&D Management, v. 39, n. 4, p. 311-316, 2009.

ERZURUMLU, S. Collaborative product development with competitors to stimulate downstream innovation. International Journal of Innovation Management, v.14, n. 4, p. 573-602, 2010.

GARDET, E.; FRAIHA, S. coordination modes established by the hub firm of an innovation network: the case of an SME Bearer. Journal of Small Business Management, v. 50, n. 2, p. 216-238, 2012.

GARRETÓN, M. A; CAVAROZZI, M.; CLEAVES, P. S.; GEREFFI, G.; HARTLYN, J. América Latina no 
Século XXI:Em direção a uma nova matriz sociopolítica. Rio de Janeiro: FGV, 2007.

GASSMANN, O.; ENKEL, E.; CHESBROUGH, H. W. The future of open innovation. R\&D Management, v. 40 , n. 3, p. 213-221, 2010.

GANN, D. "Book review - Open Innovation: The New Imperative for Creating and Profiting from Technology". Research Policy, v. 1, n. 10, p. 122-123, 2004.

GRÖNLUND, J.; SJÖDIN, D. R.; FRISHAMMAR, J. Open Innovation and the Stage-Gate Process: a revised model for newproduct development. California management review. v. 52, n. 3, p. 106-131, 2010.

GRIMALDI, M.; QUINTO, I.; RIPPA, P. Enabling Open Innovation in Small and Medium Enterprises: A Dynamic Capabilities Approach. Knowledge and Process Management, v. 20, n. 4, p. 199-210, 2013.

IBGE. Site institucional. Instituto Brasileiro de Geografia e Estatística, 2006. Disponível em: http://www. ibge.gov.br/home/. Acesso em: 11 nov. 2014.

JOHNSON, G.; SCHOLES, K.; WHITTINGTON, R.Explorando a Estratégia Corporativa. Texto e Casos. $7^{\circ}$ ed. Porto Alegre: Bookman. 2007.

KONSTI-LAAKSO, S.; PIHKALA, T.; KRAUS, S. Facilitating SME innovation capability through business networking. Creativity and innovation management, v. 21, n. 1, p. 93-105, 2012.

KIRSCHBAUM, R. Open innovation in practice. Research Technology Management, v. 48, n. 4, p. 2428, 2005.

LAURSEN, K.; SALTER, A. Open for innovation: the role of openness in explaining innovation performance among U.K. manufacturing firms.Strategic Management Journal, v. 27, p. 131-150, 2006.

LEE, S.; PARK, G.; YOON, B.; PARK, J. Open innovation in SMEs-an intermediated network model. Research Policy, v.39, p. 290-300, 2010.

LOVE, J. H.; ROPER, S.; VAHTER, P. Research notes and commentaries learning from openness: the dynamics of breadth in external innovation linkages.Strategic Management Journal, v. 35, p. 17031716, 2014.

MARIANO, E. B.; GUERRINI, F. M.; REBELATTO, D. A. do N. Análise da relação entre estrutura e desempenho de redes interorganizacionais colaborativas. Gestão Produção, v. 19, n. 3, p. 471- 479, 2012.

MCGRATH, R. G.; TSAI, M.; VENKATARAMAN, S; MACMILLAN, I. C. Innovation, competitive advantage and rent: a model and test. Management Science, v. 42, n. 3, p. 389-403, 1996.

MCIVOR, R. How the transaction cost and resource-based theories of firm inform outsourcing evaluation. Journal of Operation Management, v. 27, n. 1, p. 45-63, 2009.

MORROW, JR. J. L.; SIRMON, G. D.; HITT, A. M.; HOLCOMB, R. T. Creating value in the face of declining performance: firm strategies and organizational recovery. Strategic Management Journal, v. 28, p. 271-283, 2007.

NARULA, R. R\&D collaboration by SMEs: new opportunities and limitations in the face of globalization, Technovation, v. 24, n. 2, p. 153-161, 2004.

NEWBERT, L. S. Value, rareness, competitive advantage, and performance: a conceptual-level empirical investigation of the resource-based view of the firm. Strategic Management Journal, v. 29, p. 745-768, 2008.

NISIYAMA, E. K.; OYADOMARI, J. C. T. A busca da inovação e a cadeia de valores. Revista de Administração da UNIMEP. v.10, n.1, p. 189-214, 2012.

OLIVEIRA, R. F.; GUERRINI, F. M. Características das tipologias de redes de cooperação entre 
empresas. XXII Encontro Nacional de Engenharia de Produção,ENEGEP, 2002.

PADILLA-MELÉNDEZ, A.; AGUILA-OBRA, A. R.D.; LOCKETT, N. Shifting sands: Regional perspectives on the role of social capital in supporting open innovation through knowledge transfer and exchange with small and medium-sized enterprises. International Small Business Journal, v. 31, n. 3, p. 296-318, 2012.

PARIDA, V.; WESTERBERG, M.; FRISHAMMAR, J. Inbound open innovation activities in High-Tech SMEs: the impact on innovation performance.Journal of Small Business Management,v.50, n. 2, p. 283-309, 2012.

PITASSI, C. A virtualidade nas estratégias de inovação aberta: proposta de articulação conceitual. Revista de administração pública, v. 46, n. 2, p. 619-641, 2012.

PORTER, M. E. Vantagem competitiva:Criando e sustentando um desempenho superior. Rio de Janeiro: Campus Elsevier, 1989.

PORTER, M. E. Competição: Estratégias Competitivas Essenciais. Rio de Janeiro: Campus Elsevier, 1999.

POWELL, T. C. Competitive advantage: logical and philosophical considerations. Strategic Management Journal, v. 22, n. 9, p. 875-888, 2001.

RASERA, M.; BALBINOT, Z. Redes de inovação, inovação em redes e inovação aberta: um estudo bibliográfico e bibliométrico da produção científica no Enanpad 2005-2009 sobre inovação associada a redes. Análise, v. 21, n. 2, p. 127-136, 2010.

ROBERTSON, P. L.; CASALI, G. L.; JACOBSON, D. Managing open incremental process innovation: absorptive capacity and distributed learning. Research Policy, n. 41, p. 822-832, 2012.

RUMELT RP. How much does industry matter? Strategic Management Journal, v. 12, n. 3, p. 167-185, 1991.

SEBRAE. Site institucional. Serviço Brasileiro de Apoio às Micro e Pequenas Empresas. As micros e pequenas empresas na exportação brasileira. Disponível em: http://201.2.114.147/bds/bds.nsf/321D773E 81BA504A03256F1E004FE13A/\$File/NT0009D5EA.pdf. Acesso em: 25 fev. 2015.

SEBRAE. Site institucional. Serviço Brasileiro de Apoio às Micro e Pequenas Empresas. Desempenho exportador das micro e pequenas empresas: nota metodológica, 2012. Disponível em:

http://www.sebrae.com.br/br/pesquisa_exportacao/ notametodologica.asp. Acesso em: 11 jan.. 2015.

SENER, S.; HOBIKOGLU, E. H. Structural effect of enterprises open-closed innovation models tendencies in product output process: a study on the enterprises located in the IMES industrial estate Turkey example. Procedia - Social and Behavioral Sciences, v.99, n. 6, p. 986-996, 2013.

SILVA, G.; DACORSO, A. L. Inovação aberta como uma vantagem competitiva para a micro e pequena empresa. INMR - Innovation \& Management Review, v. 10, n. 3, p. 251-268, 1 out. 2013.

SILVA, M..; ZILBER, M. Benefícios percebidos pela adoção do processo de inovação aberta. Revista de Administração da UNIMEP, v. 11, n.3, p. 1-17, 2013.

SPITHOVEN, A.; WANHAVERBEKE, W.; ROIJAKKERS, N. Open innovation practices in SMEs and large enterprises. Small Business Economics, v. 41, n. 3, p. 537-562, 2013.

STAL, E.; NOHARA, J. J.; CHAGAS, M. de F. Jr. Os conceitos da inovação aberta e o desempenho de empresas brasileiras inovadoras. Revista de Administração e Inovação, v. 11, n. 2, p. 295-320, 2014.

TIDD, J.; BESSANT, J.; PAVITT, K. Managing Innovation: Integrating technological, market and organisational change. 2 ed. Chichester: Jonh Wiley, 2001.

TSAI, K.; WANG, J. External technology sourcing and innovation performance in LMT sectors: an 
analysis bases on the Taiwanese technological innovation survey. Research Policy, v. 38, n. 3, p. 518526, 2009.

UN, A. C.; CUERVO-CAZURRA, A.; ASAKAWA, K. R\&D collaborations and product innovation.Journal of Product InnovationManagement, v. 27, n. 5, p. 673-689, 2010.

VAN DE VRANDE, V.; JONG, J. P. J.; VANHAVERBEKE, W.; ROCHEMONT, M. (2009). Open innovation in SMEs: trends, motives and management challenges. Technovation. v. 29, p. 423-437, 2009.

VAN de VEN, A. H. V. Central problems in the management of innovation. Management Science, v. 32, n. 5, p. 550-607, 1986.

VANHAVERBEKE, W.; VAN DE VRANDE, V.; CHESBROUGH, H. W. Understanding the advantages of open innovation practices in corporate venturing in terms of real options. Creativity and Innovation Management,v. 17, n. 4, p. 251-258, 2008.

VOSSEN, R. W. Relative strengths and weaknesses of small firms in innovation. International Small Business Journal, v. 16, n.3, p. 88-94, 1998.

WESTERGREN, U. H.; HOLMSTRÖM, J. Exploring preconditions for open innovation: Value networks in industrial firms. Information and Organization, v. 22, n. 4, p. 209-226, 2012.

WIGGINS, R. R.; RUEFLI, T. W. Sustained competitive advantage: temporal dynamics and the incidence and persistence of superior economic performance. Organization Science,v. 13, n. 1, p. 82-105, 2002.

WILLIAMSON, O. E. The economics of organization: the transaction cost approach. The American Journal of Sociology, v. 87, n. 3, p. 548-577, 1981.

WOERTER, M; ROPER, S. Openness and innovation - home and export demand effects on manufacturing innovation: panel data evidence for Ireland and Switzerland. Research Policy, v. 39, p. 155-164, 2010.

WYNARCZYK, P.; PIPEROPOULOS, P.; MCADAM, M. Open innovation in small and medium-sized enterprises: an overview.International Small Business Journal, v. 31, n. 3, p. 240-255, 2013.

Submetido em: 09/02/2018

Aceito em: 30/07/2019 